\title{
RESEARCH INVOLVING ADULTS WHO LACK CAPACITY: HOW HAVE RESEARCH ETHICS COMMITTEES INTERPRETED THE REQUIREMENTS?
}

Mary Dixon-Woods, Professor of Medical Sociology, Social Science Research Group, Department of Health Sciences, 2nd floor Adrian Building, University of Leicester, Leicester, LE1 7RH, UK. Tel + 441162297262; Fax: +441162297250; Email: md11@le.ac.uk [CORRESPONDING AUTHOR]

Emma L Angell, Research Associate, Social Science Research Group, Department of Health Sciences, 2nd floor Adrian Building, University of Leicester, Leicester, LE1 7RH, UK. Email: elj1@le.ac.uk

The Corresponding Author has the right to grant on behalf of all authors and does grant on behalf of all authors, an exclusive licence (or non exclusive for government employees) on a worldwide basis to the BMJ Publishing Group Ltd, and its Licensees to permit this article (if accepted) to be published in BMJ editions and any other BMJPGL products and to exploit all subsidiary rights, as set out in our licence (bmj.com/advice/copyright.shtml) 


\section{ABSTRACT}

Aim: Two separate regulatory regimes govern research with adults who lack capacity to consent in England and Wales: the Mental Capacity Act (MCA) 2005 and the Medicines for Human Use (Clinical Trials) Regulations 2004. We conducted a service evaluation to investigate how Research Ethics Committees (RECs) are interpreting the requirements.

Methods: Using a coding scheme, assisted by qualitative software, we analysed a sample of REC decision letters where applicants indicated that their project involved adults who lacked mental capacity.

Results: Our analysis focuses on 45 letters about projects covered by the MCA and 12 letters about projects covered by the Clinical Trials Regulations. Legal requirements about involving incapacitated adults in research were not consistently interpreted correctly. Letters often lacked explicitness and clarity. Neither consent nor assent from third parties are legally valid concepts for purposes of the MCA, yet they were suggested or endorsed in 10 post-MCA letters, and there was evidence of confusion about the consultee processes. The correct terms were also not consistently used in relation to clinical trials. There was potential for inappropriate use of terms such as 'relative' to exclude people who are eligible to be consulted.

Conclusions: Unless the correct terms and legal concepts are used in research projects, there is potential for confusion, and exclusion of people who are eligible to be consulted about involvement of adults who lack capacity. There is a need for improved clarity, explicitness, and accuracy when submitting and reviewing applications for ethical review of research in this area. 


\section{INTRODUCTION}

There are now two separate regulatory regimes governing research with adults who lack capacity to consent in England and Wales: the Mental Capacity Act 2005 and the Medicines for Human Use (Clinical Trials) Regulations 2004. The Mental Capacity Act (MCA) $2005{ }^{1}$ came into force in England and Wales in two stages in April and October 2007. The MCA offers, for the first time, a comprehensive legal framework for decision-making on behalf of adults (aged 16 years and over) who lack capacity to make decisions for themselves. One of its novel features is a set of requirements governing how incapacitated adults can be involved in research. The Act thus clarifies a long-running area of concern for professionals and researchers, though it excludes clinical trials of investigational medicinal products (CTIMPs). ${ }^{2}$

CTIMPs involving adults who lack capacity are regulated separately by the Medicines for Human Use (Clinical Trials) Regulations 2004. ${ }^{3}$ Though there are many similarities between the two regulatory regimes (Figure 1), there are important differences. In particular, CTIMPs require consent from a legal representative; non-CTIMPs require consultation with a consultee.

The National Research Ethics Service (NRES) has designated around 30 RECs to receive applications under the MCA, and these RECs have been given special training. Applications to conduct CTIMPs must also be considered by separate specialist ('recognised') RECs. RECs issue decision letters in response to applications, which may give one of three opinions (Box 1). It has been suggested that researchers should work closely with Research Ethics Committees (RECs) to ensure that the stipulations of the MCA are followed, ${ }^{4}$ but little is known about how RECs have interpreted its requirements or those of the CTIMPs regulations in relation to incapacity. In this paper, we report a service evaluation of how RECs have responded to legal requirements governing adults who lack capacity to consent to research.

\section{SERVICE EVALUATION: METHODS}

When applying for REC approval, researchers are required to declare (by ticking a box on the electronic application form) whether they plan to include adults unable to consent for themselves in their research. As part of a service evaluation commissioned by NRES, we analysed anonymised REC decision letters about applications where this box had been ticked. NRES advised that ethics committee approval was not required for our project because of its status as service evaluation. Researchers' applications themselves could not be analysed without permission from the applicants and were not included in the analysis.

We were interested in applications involving adults who lacked mental capacity both before the publication of the MCA in April 2005 and after its implementation in October 2007. We therefore constructed two sampling epochs: a pre-MCA epoch ( $1^{\text {st }}$ March 2004 - when the database began - to $1^{\text {st }}$ April 2005) and a post-MCA epoch ( $1^{\text {st }}$ October 2007 to $28^{\text {th }}$ February 2008 - when our service evaluation began). For purposes of this analysis, because we were interested in RECs' interpretation of the Clinical Trials Regulations and the Mental Capacity Act, we included CTIMPs projects from both epochs, but for non-CTIMPs projects we focused only on letters issued after the implementation of the MCA.

Our inclusion criteria were that: the application indicated that the research would involve adults who lacked capacity; it was a full application for a new study (not a site-specific assessment or a protocol amendment for example); the application had been submitted to a REC in England or Wales; and the application had received a provisional or unfavourable opinion. Our exclusion criteria were: the 
application had received a favorable opinion at first review (thus providing little or no suitable text for analysis); the application was withdrawn, deemed to be outside remit; and the applicants had ticked a box indicating that the research involved children under 16, prisoners, young offenders, healthy volunteers, those who could be considered to have a particular dependent relationship with the investigator, or other vulnerable groups. Although some RECs have recently been assigned as 'specialist' RECs to review applications involving adults with involving incapacity, this project sampled letters regardless of REC status.

Using the NRES database, we identified 308 applications meeting our criteria in the pre-MCA epoch, while there were 65 applications meeting our criteria in the post-MCA epoch. From each epoch, we randomly selected (using a table of random numbers) 50 applications where the applicants indicated that their research involved adults who lacked mental capacity and met the other inclusion/exclusion criteria. Applications where no letter had been uploaded to the database were excluded at this stage. As letters were unavailable for 14 applications in the post-MCA epoch and a further application was ineligible because of reasons relating to the date, all 50 remaining applications were included - no further sampling was possible.

To conduct the analysis, we applied a coding scheme developed in previous studies by using ethnographic content analysis and traditional content analysis. ${ }^{567}$ This coding scheme allows reliable categorisation of the kinds of issues that RECs raise and how the RECs propose these be resolved. The coding scheme was further refined in this project to address new themes identified in this project, specifically in relation to legal issues pertaining to mental capacity. Using these methods allowed us to combine powerfully a systematic and explicit approach to coding of data with a commitment to interpretation and reflexivity. QSR N6 software was used to assist in the analysis.

\section{RESULTS}

The post-MCA epoch included 45 applications covered by the MCA, which we term the 'MCA letters' Twelve applications relating to CTIMPs were included in our sample: seven from the pre-MCA epoch and five from the post-MCA epoch. We term these the 'CTIMPs letters'.

\section{THE MCA LETTERS}

The 45 post-MCA letters had been considered by 21 different RECs. Most (38/45) of these letters gave applicants a provisional opinion, meaning that some further modifications or response was required before a final decision could be issued. Of these initially provisional applications, all except one (for which the decision was unavailable at the time of analysis) were subsequently given a favourable opinion. Seven of the 45 applications were issued with an unfavourable opinion at first review. The unusually high rate of unfavourables $(15 \% \text {, compared with the normal rate of } 8 \%)^{7}$ might be due to researchers' unfamiliarity with the MCA regulations or the stringent implementation of the regulations by RECs, but with the small numbers involved it is difficult to be certain about whether this is a genuine difference in rates of unfavourable opinions.

In 25 of the 45 letters, RECs explicitly showed that they were concerned with ensuring compliance with the Mental Capacity Act. In two letters, RECs explicitly declined to approve the proposed project for purposes of the MCA. In 11 letters, they were concerned with processes of conducting research involving adults who lack capacity, including how capacity could be assessed.

"The Committee raised concern about obtaining consent at a time of distress and in a population with high levels of illiteracy. The Committee would like clarification on how this will be dealt with 
and how lack of capacity to consent would be assessed." (Letter A11, non-CTIMP, provisional opinion then favourable)

However, despite their evident engagement with issues of mental incapacity, RECs were not consistently fully explicit or correct in their interpretation of MCA requirements. We judged that 15 of the 21 RECs did not provide completely clear, explicit or correct advice about the MCA, particularly in relation to justifications for including people who lack capacity and the consultee process.

\section{Justification for including people who lack capacity}

Under the MCA, research involving, or in relation to, a person lacking capacity may be lawfully carried out only if an "appropriate body" (normally a REC) approves it. RECs must judge the research against set criteria (Box 2). In 15 of the 45 MCA letters, RECs raised issues about whether people with incapacity to consent should be included or excluded from research. Only a small proportion (8/45) of letters commented explicitly on the justification for including people with capacity in the research, and a smaller proportion (5/45) commented explicitly on whether the research would benefit people with incapacity. These small numbers may well be because the applicants provided sufficient information to reassure RECs, and RECs may therefore have felt no need to comment. However, given the requirements of the MCA in relation to these matters, and the need for accountability, it is worth considering whether RECs should be consistently explicit in endorsing the applicants' proposals - for example by stating that "the REC considered and accepted your explanation for why the involvement of people with incapacity was justified for this project and that it meets the requirements for benefits and risks of research under the Mental Capacity Act 2005."

\section{Consultee process under the Mental Capacity Act}

The MCA requires the researcher to take steps to identify a personal consultee who has a role in caring for the person who lacks capacity or is interested in that person's welfare, but is not doing so for remuneration or acting in a professional capacity. ${ }^{8}$ In situations where there is no personal consultee available, the researcher may identify a nominated consultee (e.g. a General Practitioner) who is not connected with the research. The researcher must provide the consultee with information about the research project, and ask for advice on whether the person in question should take part in the project, and the consultee's opinion regarding the person's wishes and feelings about taking part if the person had capacity. Importantly, the consultee gives advice, not consent, as under the MCA there is no 'consent' representing the presumed will of the participant in law.

There was some evidence from RECs' comments in letters that applicants were not always clear about what was required to comply with the MCA. For example, applicants' proposals to use 'proxies' were rejected by RECs in two letters, since proxies are not recognised by the MCA. Six letters also corrected applicants who proposed to seek 'assent' from relatives/carers, as the categories of 'assent' or 'assenter' are not legally recognised by the MCA .

Correcting applicants: "A separate form should be provided for consultees to sign. It should be similar to a consent form, but cannot use words such as assent or consent" (Letter A60, nonCTIMP, provisional opinion then favourable)

However, there was considerable evidence that RECs themselves were not consistently getting it right: 27 of the 45 letters talked about the consultee process, but we judged that only one of these gave advice that could be considered entirely correct and explicit.

Ten letters appeared to be giving inappropriate advice in relation to issues of assent/consent, sometimes by accepting or endorsing what applicants themselves were proposing, and sometimes by actively requesting changes. Three letters (all from the same REC) incorrectly advised applicants to obtain 
consent from relatives/carers. In seven letters (three from one REC and the remainder from four different RECs), applicants were inappropriately advised to obtain assent.

Incorrectly advising consent: "Relative assent form. The form should be rewritten as it does not take into account the changes brought about by the Mental Capacity Act. It would be better to describe the sheet as 'Personal Consultee Consent Form' [...]" (Letter A27, non-CTIMP, provisional opinion then favourable)

Incorrectly advising assent: "please change consent to assent if being given by one person on behalf of another" (Letter A47, non-CTIMP, provisional opinion then favourable)

Eighteen of the 45 letters (four from one REC, three each from two other RECs, and the remaining eight from different RECs) gave advice that might be considered ambiguous, lacking in clarity, misleading or contradictory in relation to the type of person who could be consulted. For example, we found one letter that referred to obtaining assent from legal representatives, even though the term 'legal representative' can only be used in relation to CTIMPs. Letters frequently used terms such as 'carer' without making clear whether they were referring to paid or unpaid carers. Terms such as 'relative' and 'next of kin' were also used, and could give the misleading impression that only people related to the patient could be consulted, which is not what is intended by the MCA. Letters did not consistently use terms such as 'personal' and 'nominated' consultee explicitly or correctly. One letter, for example, described using a 'professional' consultee rather than a 'nominated' consultee. Only three letters used the term 'nominated'. In three other letters (all from the same REC), applicants were incorrectly advised that paid carers cannot act as consultees for the patient, failing to make clear that while paid carers may not be personal consultees, they may act as nominated consultees as long as they are unconnected with the research project.

Ambiguity about carers acting as consultees: "The Committee requested reassurance that the researchers are aware that paid carers are unable to act as consultees for patients in accordance with the Mental Capacity Act." (Letter A35, non-CTIMP, provisional opinion then favourable)

\section{Applications to conduct clinical trials of investigational medicinal products}

Of the 12 CTIMPs-related letters in our sample, 10 issued provisional opinions (followed by favourable), and two issued unfavourable opinions at first review. The Clinical Trials Regulations implement the European Union Clinical Trials Directive, ${ }^{9}$ which specifies that people who are incapable of giving legal consent to clinical trials should be given special protection. A medicinal product should only be administered to someone who lacks capacity in a trial if there are grounds for expecting that the product will produce a benefit to the person that outweighs the risks, or has no risk. The trial should relate directly to a life-threatening or debilitating clinical condition from which the person suffers. In these respects the Regulations therefore share similarities with the Mental Capacity Act. However, there are some important distinctions. Rather than using consultees, the Regulations require that informed consent be obtained on behalf of the patient from the patient's legal representative. This legal representative must have no connection to the conduct of the trial, and may be a personal legal representative (e.g. a relative or friend) or a professional legal representative, who should be approached if no personal legal representative is available and should be nominated by the relevant care provider (e.g. NHS trust).

There was evidence in the letters we looked at that RECs were giving advice about seeking consent from legal representatives for CTIMPs, but did not consistently give fully correct or explicit advice. In four letters where advice was given about the consent process, the advice was in some way lacking in clarity or misleading. In two letters, RECs advised applicants (or endorsed applicants' proposals) to seek assent rather than consent. No letter correctly and explicitly made clear the role and distinctions 
between the personal and professional legal representative, instead referring to relatives, next of kin, carers and so on. Again, inappropriate views about who is entitled to consent on behalf of a participant may thus be inadvertently encouraged.

Reference to next of kin: "A consent form for next of kin should be produced if it was the intention to recruit adults without capacity to consent for themselves." (Letter A44, CTIMP, provisional opinion then favourable)

\section{Sources of mental incapacity mentioned by RECs}

Many REC letters about both CTIMPs and non-CTIMPs projects referred to different conditions that might result in people losing capacity to consent to research. These included dementia/Alzheimer's, illiteracy, terminal illness, learning disabilities/cognitive impairment and unconsciousness. But we found no letters that commented on involving people with mental illnesses (such as schizophrenia or psychosis) that might interfere with capacity.

\section{CONCLUSIONS}

Both RECs and applicants need to be very clear about legal requirements in relation to involvement of adults who lack capacity in research. The law in this area is complex and confusing, with two different regimes with some similarities and some differences. Our analysis suggests that in the period immediately following the implementation of the Mental Capacity Act, RECs were not always giving advice to applicants that was consistent with the provisions of the Act. There is a need for consistent, accurate, and explicit use of the terms used by the MCA in REC letters and in applications for REC approval. Neither consent nor assent from third parties are legally valid concepts for purposes of the MCA, yet it was suggested or endorsed in 10 of the 45 letters we examined. There was also evidence of confusion about the consultee process. The terms 'personal consultee' and 'nominated consultee' need to be used consistently and explicitly, and both applicants and RECs need to be aware that paid carers can act as nominated consultees. For CTIMPs, there is also need for RECs and applicants to recognise that 'assent' is not the appropriate term, and that the terms 'personal legal representative' and 'professional legal representative' should be explicitly and consistently used. Terms such as 'relative' and 'next of kin' are to be avoided, as they are misleading and may exclude people who are eligible to be consulted.

We would also suggest that RECs should explicitly accept or reject applicants' proposals regarding why the involvement of people with incapacity is justified for each project, and whether the project will provide knowledge relevant to the condition (or similar condition) affecting the participant, or have potential benefit for the participant without disproportionate risks.

The National Research Ethics Service, who commissioned this service evaluation, is already taking steps to correct the problems identified by our analysis and there is an ongoing training programme on the MCA for RECs. It is important that applicants are also aware of the legal requirements governing the involvement of adults who lack capacity to consent in research; they should prepare their applications accordingly and be well informed in their responses to REC decision letters. 
Acknowledgements: We thank the National Research Ethics Service for funding this analysis. We thank our colleagues Professor Richard Ashcroft and Noelle Robertson for helpful comments.

Contributorship: MDW led the design, obtained funding, supervised the analysis, and wrote the first draft of the paper. EA conducted the analysis and contributed to writing the paper. Both authors approved the final version.

Guarantor: MDW is the guarantor for this paper.

Competing interests: All authors declare that the answer to the questions on your competing interest form - http://resources.bmj.com/bmj/authors/checklists-forms/competing-interests - are all No and therefore have nothing to declare. NRES funded this analysis but had no role in designing or conducting the study, writing of the paper, or the decision to submit. 
A 'favourable' opinion means that an application is approved without further amendments; these constitute $\sim 17 \%$ of decisions made by RECs at first consideration of an application.*

'Provisional' opinions constitute $\sim 66 \%$ of decisions at first review, and require applicants to make a response addressing issues raised in the letter before a final opinion can be issued. The final opinion may be either favourable or unfavourable.

An 'unfavourable' opinion ( $\sim 8 \%$ of all submissions) at first review amounts to a rejection.

RECs may also decide that applications are 'outside remit'.

Some applications are withdrawn $(\sim 10 \%$ before review and $\sim 2 \%$ after a provisional opinion has been issued).

*Data based on the period October 2005-March 2008 from the NRES website and refer to all applications. 
Box 2: Criteria for approval of a research project involving adults who lack mental capacity

The REC may only approve the research project if the research is linked to:

an impairing condition that affects the person who lacks capacity or

the treatment of that condition

and

there are reasonable grounds for believing that the research would be less effective if only people with capacity are involved, and

the researchers have made arrangements to consult carers and to follow the other requirements of the Act.

Research involving adults who lack capacity must meet one of two requirements:

the research must have some chance of benefiting the person who lacks capacity, and this benefit should be in proportion to any burden of taking part, or

the aim of the research must be to provide knowledge about the cause of, or treatment or care of people with the same impairing condition or a similar condition. If this is the case, the risks to the person with incapacity must be negligible, there must be no significant interference with the freedom of action or privacy of the person, and nothing unduly invasive or restrictive may be done.

Department for Constitutional Affairs. (2007). Mental Capacity Act 2005: Code of practice. London: The Stationery Office. 

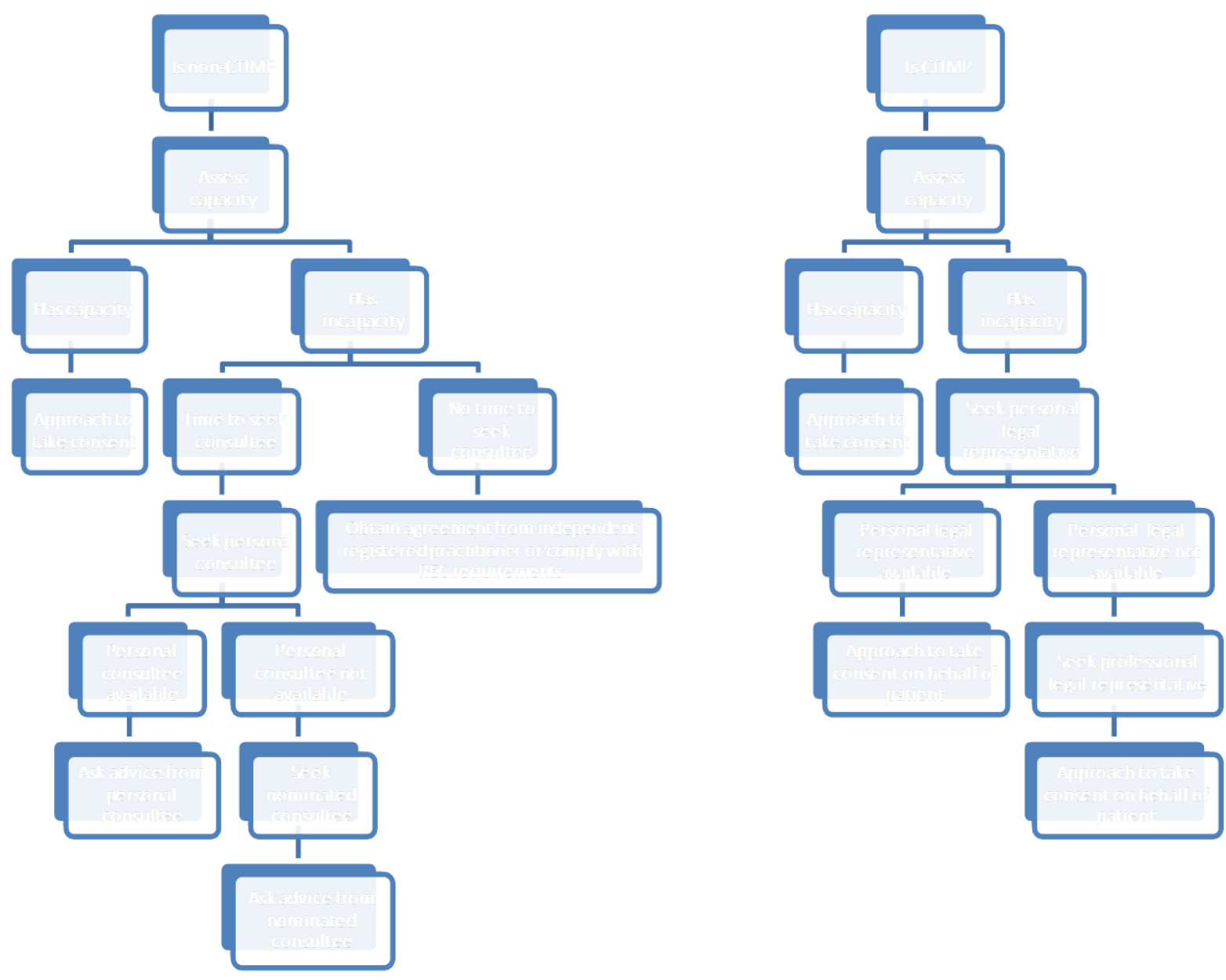

${ }^{1}$ Mental Capacity Act 2005 http://www.opsi.gov.uk/ACTS/acts2005/ukpga_20050009_en_1

${ }^{2}$ Liddell K, Bion J, Chamberlain D, Druml C, Kompanje E, Lemaire F, Menon D, Vrhovac B, Wiedermann CJ. Medical research involving incapacitated adults: implications of the EU clinical trials directive 2001/20/EC. Medical Law Review 2006; 14: 367-417

${ }^{3}$ Statutory Instrument 2004 No 1031. The Medicines for Human Use (Clinical Trials) Regulations 2004 http://www.opsi.gov.uk/si/si2004/20041031.htm

${ }^{4}$ Ludman AJ. Mental Capacity Act: how research is affected. BMJ 2008; 336: 405

5 Dixon-Woods M, Angell E, Ashcroft RE, Bryman A. Written work: the social functions of Research Ethics Committee letters. Soci Sci Med 2007; 4: 127-8

${ }^{6}$ Angell EL, Jackson CJ, Ashcroft RE, Bryman A, Windridge K, Dixon-Woods M. Is "inconsistency" in Research Ethics Committee decision-making really a problem? An empirical investigation and reflection. Clinical Ethics 2007; 2: 92-99

${ }^{7}$ Angell E, Bryman A, Ashcroft R, Dixon-Woods M. An analysis of decision letters by research ethics committees: the science/ethics quality boundary examined. Qual Saf Health Care 2008; 17:131-136

${ }^{8}$ Department of Health (2008) Guidance on nominating a consultee for research involving adults who lack capacity to consent. 
http://www.dh.gov.uk/en/Publicationsandstatistics/Publications/PublicationsPolicyAndGuidance/DH_083 131

${ }^{9}$ Directive 2001/20/EC of the European Parliament and of the Council of 4 April 2001 on the approximation of the laws, regulations and administrative provisions of the Member States relating to the implementation of good clinical practice in the conduct of clinical trials on medicinal products for human use. Official J Eur Commun 2001;L121:34-44 